\title{
IT in the Chinese Healthcare Industry
}

By: Nir Kshetri

Kshetri, Nir (2013). "IT in the Chinese Healthcare Industry”, IEEE IT Professional, 15(1), 1215. http://doi.ieeecomputersociety.org/10.1109/MITP.2013.14

(c) 2013 IEEE. Personal use of this material is permitted. Permission from IEEE must be obtained for all other users, including reprinting/ republishing this material for advertising or promotional purposes, creating new collective works for resale or redistribution to servers or lists, or reuse of any copyrighted components of this work in other works.

\author{
Abstract: \\ China is leveraging the cloud and mobile computing to enable doctors and other healthcare \\ professionals to collaborate, share information, and more easily access reports, scans, EMRs, lab \\ reports, prescriptions, and medical histories. China is also garnering government support and \\ help from various foreign companies. Here, the author reviews some of these efforts as he \\ examine the current state and future directions of the Chinese healthcare industry.
}

Keywords: hospitals | healthcare industry | China | Chinese healthcare industry | information technology | emerging markets | cloud computing | mobile computing | educational institutions Article:

The Chinese healthcare industry is undergoing rapid informatization, recording double-digit growth after massive investments from the government, hospitals, venture capitalists, and private equities. The industry spent approximately US $\$ 850$ million on IT in 2007, but it's expected to reach $\$ 2.4$ billion in 2013 and then continue to grow annually by 20 percent. 1 Despite this growth, the market is suffering from fragmentation, incompatibility issues, and rural-urban discrepancies. As hospitals and doctors' offices transition over to electronic health records (EHR) and electronic medical records (EMR), the industry hopes to improve its efficiency, reach, effectiveness, and competitiveness.

As part of this effort, China is leveraging the cloud and mobile computing to enable doctors and other healthcare professionals to collaborate, share information, and more easily access reports, scans, EMRs, lab reports, prescriptions, and medical histories. China is also garnering government support and help from various foreign companies. Here, I review some of these efforts as I examine the current state and future directions of the Chinese healthcare industry.

The Challenges

China has made substantial progress in implementing its planned hospital information system (HIS). According to the Chinese Ministry of Health, in 2008, 80 percent of hospitals had implemented the HIS. 2 Progress has been slower on the EMR front, however. Many hospitals 
still handle patient data on paper, making it difficult to access a patient's history and determine the appropriate treatment. Community clinics and hospitals are also plagued by problems such as outdated IT equipment, a lack of collaboration, and a shortage of IT professionals. Consequently, Chinese hospitals and clinics are struggling to efficiently meet the growing needs of patients.

Furthermore, China's healthcare is an "information island." Although many large hospitals completed IT initiatives in the mid 2000s, their information systems are fragmented and incompatible with other departments, inhibiting communication and data sharing. One reason for the slow EMR diffusion is the lack of a single standard.

Another barrier is healthcare professionals' low propensity to adopt ICT. In a recent survey, 66 percent of respondents said that they think technology and telecommunications companies and consumers in China are more interested in mobile healthcare (m-health) than doctors are. 3 Furthermore, wide rural-urban discrepancies exist with respect to health and healthcare resources, leading to different patterns of development and thus varying levels of sophistication in the healthcare IT market. In response to the government's call for wider IT use in the healthcare industry, Beijing, Shanghai, and developed cities in the East and South coast have established a unified healthcare IT system and started healthcare IT trials. The government's plan to construct new facilities must now focus on the less developed regions.

Despite these challenges, the IT and healthcare industries have already adopted some of the necessary technologies to quickly move forward with China's informatization of the healthcare sector.

Health Cloud Deployment

The cloud can transform the Chinese healthcare industry and improve patients' quality of care by generating horizontal and vertical collaboration among hospitals and other organizations. It can provide platforms for storing, sharing, and synchronizing patient profiles and for enhancing the efficiency of hospital management. Chinese healthcare organizations are thus rapidly adopting the cloud to enhance their competitiveness.

The Shanghai government deployed China's first "health cloud" in the Zhabei district, integrating different clouds for "healthcare," "community health," and "district-wide health." Likewise, the city of Dongying plans to add healthcare services to the cloud to centralize patients' records and make them more readily available. The Shanghai Pudong Health cloud hosts medical records of patients in hospitals and community health centers. The information lets patients visit outpatient or small regional clinics instead of specialized doctors located farther away. 4 Similarly, in a hospital in the city of Jinan, machines provide patients with automatic services. Anyone visiting the hospital receives a card, which is used to register, pay, and receive test results.

Mobile Healthcare 
M-health is the Chinese healthcare informatization industry's sweet spot. The majority of China's population doesn't own a PC, but by the first quarter of 2012, there were 1.02 billion mobile phone users, and 80 percent of doctors and consumers in China view widespread adoption of mhealth as "inevitable." 3 M-health would improve doctors' interactions with hospitals and patients, and mobile carriers hope to capitalize on this opportunity.

China Telecom has launched m-health pilot projects in over 200 hospitals 5 and has teamed up with Cambridge University to research m-health informatization. China Unicom has developed a "cloud computing storage" service platform to collect, transmit, and retrieve healthcare data. The company has also developed systems for m-health rescue positioning and the real-time transfer of vital signs, such as heart beat, breathing rate, temperature, and blood pressure. 5

Government Initiatives

It's important to note the government's role in making healthcare a key focus area for ICT investment. The Health Care Reform Leading Group was established in 2006, and in 2009, the government announced a national health reform plan and made a commitment to spend US\$124 billion during 2009-2011. As a component of the reforms, 22 provinces and municipalities in rural regions were selected to test a system for using and managing EMRs. By the end of 2010, approximately 100 hospitals in those provinces and municipalities had launched EMR pilot trials. 6

Similarly, China's 12th Five-Year Plan has focused on healthcare reform. The State Council issued the Implementation Plan on Deepening the Reform of Healthcare System in 2011-2015, which emphasized the importance of IT implementation for enhancing efficiency as well as service standards. 5 Approximately 2,000 small and mid-sized hospitals benefited from the special funds provided by the government to support the construction and upgrade of IT systems.

\section{Support from Foreign Companies}

Major global suppliers of healthcare IT solutions, devices, and services are also hoping to capitalize on the huge potential of emerging markets such as China. Consequently, they're partnering with local industry and collaborators to provide healthcare services.

IBM

IBM is a major foreign player in the Chinese healthcare IT market. In 2009, IBM opened a Healthcare Industry Solution Lab in Beijing for hospitals and rural medical cooperatives.

With the help of IBM, the Guang Dong Hospital of Traditional Chinese Medicine, China's largest hospital with approximately four million patient visits a year, has implemented a suite of healthcare data-sharing and analytics technologies, known as the Clinical and Health Records Analytics and Sharing (CHAS) system. CHAS combines the strengths of traditional Chinese medicine (TCM) with those of modern Western medicine (MWM). It enables the sharing of 
EMRs, which incorporate both TCM and MWM data across the hospital network, and is thus expected to help the hospital standardize its patient records and assess TCM treatments' effectiveness. 7

In another high-profile project, IBM and China's Range Technology have constructed a state-ofthe-art, 6.2 million square foot Cloud Center in Langfang city. The data center will provide services, including EMR services. Range Technology will also provide Hebei Province with the advanced capabilities to conduct healthcare projects, particularly those focused in implementing EMRs. 8 Hebei was among the 22 provinces and municipalities selected for the public hospital reforms pilot.

Likewise, a collaboration of IBM and China's information services provider Yi Lian Zhong (YLZ) involves building a cloud platform for YLZ to connect 300 million people with various networks, including those of medical institutions.

Cisco

Following the 2008 earthquake of Wenchuan in Sichuan Province, Cisco formed a public-private partnership with the Chinese government and launched a corporate social responsibility program: "Connecting Sichuan." A major goal of the program was to apply ICTs to improve the quality and capacity of healthcare in Sichuan and to bridge the urban-rural healthcare gap. It follows the guidance of the "Healthy China 2020" initiative and is a pilot program for improving healthcare services through collaboration and the cloud.

The "Wenchuan Model" demonstrates how informatization promotes collaboration and helps integrate hospital resources. Wenchuan People's Hospital (WPH), a secondary-level hospital, evolved as a "center of collaboration" in the region and played a key role in integrating primaryand-tertiary-level healthcare providers. The healthcare collaboration technology and cloud platform allowed doctors at WPH to receive guidance from experts at Sichuan People's Hospital and West China Hospital. Doctors at WPH provide support to the 13 township health centers in Wenchuan. 9

\section{AT\&T}

In November 2011, AT\&T and China Telecom signed a deal that would let each other share infrastructures in the US and China. The Chinese healthcare industry is likely to benefit from AT\&T's experience in providing a cloud-based service for medical imaging and information management, supporting access, storage, and sharing of medical images.

\section{Microsoft}

Microsoft is working with Chinese hospitals and healthcare IT service providers. In 2009, it formed a partnership with the Affiliated Hospital of the Medical College of Qingdao University 
(QDUMH). The 1,995 bed QDUMH deployed the Microsoft Amalga HIS, which integrates the management of data, including EMR, patient, bed-assignment, and laboratory data. 10

In 2010, Microsoft and China's iSoftStone Information Technology signed an agreement to introduce Microsoft HealthVault technology to China ( www.microsoft.com/enus/news/press/2010/oct10/10-29mshealthvaultpr.aspx). HealthVault is a personal health application platform to store and manage health information in a personal, online account. The project initially focused on the city of Wuxi. The Microsoft-iSoftStone relationship envisioned citizens connecting to health systems and services run by government agencies, hospitals, pharmacies, and fitness facilities.

Dell

Dell has significant operations in healthcare IT markets of emerging economies such as India, China, and Brazil. Dell offers consulting services to several Chinese hospitals and is also working with several province governments in China on healthcare initiatives. 11 The company has partnered with China Telecom to standardize EHR, which involves working with hospitals throughout the country.

China's experience indicates that the development of indigenous technologies is an important trigger for the growth of the healthcare ICT industry and market. A complete reliance on foreign multinationals for all hardware, software, and service needs can lead to an incomplete healthcare ICT ecosystem. Foreign multinationals might lack the capability or willingness to develop products or services that can meet the unique needs of emerging markets.

The success of the healthcare industry's informatization in an emerging market also hinges on having well-developed systems, components, and processes that are interoperable. This will require intra- and interorganizational technological collaboration and other standardization initiatives and activities in the field of healthcare technologies.

Another lesson is the importance of the government's healthcare vision in encouraging participation from foreign and domestic companies. Policy makers must act to align incentives to develop the healthcare ICT industry. The active contribution of Cisco and other companies to the Chinese government's vision indicates that adequate public policies can play a major role in addressing the problem of rural-urban discrepancies in emerging economies' healthcare informatization.

Finally, other emerging economies are likely to benefit from Chinese companies' expertise and experience in healthcare IT. Chinese companies such as Huawei, China Telecom, and China Unicom can easily adapt the business models used in their domestic markets for other countries. Their ability to develop relevant products and deliver value in the domestic market will likely lead to a competitive advantage in other emerging economies and global markets. 


\section{References}

1. "Innovative IT to Transform the Healthcare Industry: Microsoft Builds 'Information Superhighway' for Healthcare," Microsoft, 1 Aug. 2008;

www.microsoft.com/china/ard/en/innoforuminnoforum_12.mspx.

2. B. Zhou, "Healthcare in China: At a Critical Transition Phase," Dell, 23 Aug 2011;

http://en.community.dell.com/dell-blogs/health-care/b/washington-

report/archive/2011/08/23healthcare-in-china-at-a-critical-transition-phase.aspx.

3. R. Ling and J. Xiao, "China Could Be a Fertile Ground for mHealth Applications," PriceWaterhouse-Coopers, 2012;

www.pwccn.com/webmedia/doc634828881885276267_healthcare_mhealth_2012.pdf.

4. M. Chua, "Huawei Hinges Cloud Dreams on Partners," Frost.com, 27 Oct. 2010; www.networksasia.net/contenthuawei-hinges-cloud-dreams-partners.

5. "China's Mobile Healthcare Industry to Witness Two-Digit Growth," TMCnet, 28 Mar. 2012; http://callcenterinfo.tmcnet.com/news/2012/03/286221092.htm.

6. "China: Country Health Information Profiles," World Health Organization, 2011, www.wpro.who.int/countries/chn5CHNpro2011_finaldraft.pdf.

7. L. Chao, "IBM Works on China's Medical Records," Wall Street J.,31 Aug. 2012;

http://online.wsj.com/articleSB10001424052748703369704575462653035645256.html?reflink= barrons_redirect.

8. C. Babcock, "IBM to Build Massive China Cloud Data Centers, Information Week,26 Jan. 2011; www.informationweek.com/hardware/data-centers/ ibm-to-build-massive-china-clouddata-ce 229100310.

9. "Cisco and Peking University Jointly Release the Impact Assessment Report of 'Connecting Sichuan' Healthcare Program," Cisco.com, 11 Apr. 2012; http://newsroom.cisco.compressrelease-content?type=webcontent $\&$ articleId=782178.

10. "Microsoft and the Affiliated Hospital of the Medical College of Qingdao Enter into Partnership to Improve Healthcare through Software Innovation," Microsoft, 27 Apr. 2009.

11. A. Shah, "Dell Traverses China Challenges to Expand Services Unit, IDG News Service," PC World,14 Oct. 2011;

www.pcworld.com/article/241964dell_traverses_china_challenges_to_expand_services_unit.htm $\underline{1 .}$ 